POS PROCEEDINGS

\title{
Search for exotic Higgs-boson decays in events with at least one photon, missing transverse momentum, and two forward jets produced in $\sqrt{s}=8 \mathrm{TeV} \mathrm{pp}$ collisions with the ATLAS detector
}

Catrin Bernius* on behalf of the ATLAS Collaboration

New York University

E-mail: Catrin.Berniusecern.ch

\begin{abstract}
A search is performed for Higgs-boson decays to neutralinos and/or gravitinos in events with at least one photon, missing transverse momentum and two forward jets, a topology where vector boson fusion production is enhanced. The analysis is based on a dataset of proton-proton collision data taken at $\sqrt{s}=8 \mathrm{TeV}$ delivered by the Large Hadron Collider and recorded with the ATLAS detector, corresponding to an integrated luminosity of $20.3 \mathrm{fb}^{-1}$. The observation is consistent with Standard Model expectation and upper limits are set on the production cross section times branching fraction of the Higgs-boson to decay to neutralinos and/or gravitinos.
\end{abstract}

The European Physical Society Conference on High Energy Physics

22-29 July 2015

Vienna, Austria

${ }^{*}$ Speaker. 


\section{Introduction}

The presented analysis [1] studies exotic Higgs decays to supersymmetric (SUSY) particles which could explain the Higgs mass and address the hierarchy problem using the full $20128 \mathrm{TeV}$ $p p$ dataset recorded by the ATLAS detector [2] at the LHC, corresponding to an integrated luminosity of $20.3 \mathrm{fb}^{-1}$. Two models are considered. The gauge mediated supersymmetric breaking (GMSB) model which predicts a Higgs-boson decay to a nearly massless gravitino $\tilde{G}$, the lightest SUSY particle (LSP) and a neutralino $\tilde{\chi}^{0}$ (next-to-lightest SUSY particle, NLSP), with the neutralino decaying to a photon and a gravitino, leading to a photon $\gamma$ and missing transverse momentum $E_{\mathrm{T}}^{\text {miss }}$ signature. The second model considered is the Next-to-Minimal SUSY Model (NMSSM) which also predicts a $\gamma+E_{\mathrm{T}}^{\text {miss }}$ signature with the Higgs decaying to the LSP $\tilde{\chi}_{1}^{0}$ and the NSLP $\tilde{\chi}_{2}^{0}$, which then decays to a photon and a LSP $\tilde{\chi}_{1}^{0}$. The decays chains occur when $m\left(\tilde{\chi}_{1}^{0}\right)$ and $m\left(\tilde{\chi}_{2}^{0}\right)$ are between $m_{h / 2}$ and $m_{h}$, if they are smaller than $m_{h / 2}$, the decay chains lead to a di-photon plus $E_{\mathrm{T}}^{\text {miss }}$ signature. By considering vector boson fusion (VBF) production, the Higgs-boson is produced along with two jets. The properties of the jets and effect on the Higgs decay products can then be used to reduce backgrounds and define control regions to measure backgrounds using the data. The diagrams for the decays of these two models are shown in Figure 1. In the following, only a brief summary of the analysis is presented, a more detailed description can be found in [1].

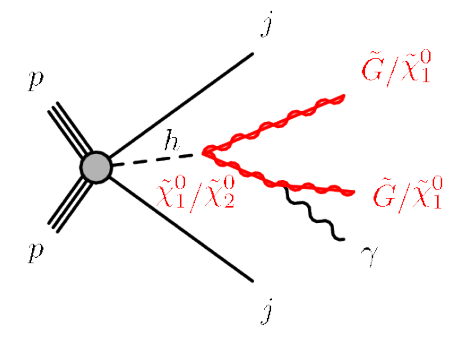

(a)

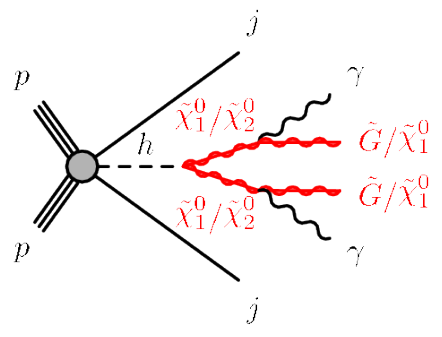

(b)

Figure 1: Diagrams for the production and decay of the Higgs-boson leading to the (a) $\gamma+E_{\mathrm{T}}^{\text {miss }}+$ $j j$ and (b) $\gamma \gamma+E_{\mathrm{T}}^{\mathrm{miss}}+\mathrm{jj}$ final state.

\section{Monte Carlo Simulation and Event Reconstruction}

The Monte Carlo (MC) simulation for the signal and background processes is described in detail in [1]. The propagation of particles in the detector is simulated using GEANT4 [3] within the full ATLAS detector simulation [4] for all MC samples except for the signal, $Z(\rightarrow v v) \gamma, W(\rightarrow l v) \gamma$ and $W(\tau \nu)$ samples for which fast simulation based on a parametric response of the calorimeters is used [5]. The effect of multiple pp collisions from the same or nearby bunch crossings (in-time or out-of-time pileup) is included in the MC samples by overlaying minimum-bias events onto the hard-scatter events. The MC samples are reconstructed in the same way as the data.

The event is reconstructed by identifying and reconstructing photons, electrons, jets and missing transverse momentum. This is described in detail in [1]. 


\section{Analysis Strategy and Event Selection}

A cut-and-count experiment is performed and the total numbers of expected background and signal events in the control and signal regions are used to set limits on $\sigma / \sigma_{S M} \times \mathrm{BF}(h \rightarrow \mathrm{NLSP}$ + LSP). The event is selected when the combined $\gamma+E_{\mathrm{T}}^{\text {miss }}$ trigger with a photon threshold of 40 $\mathrm{GeV}$ and a $E_{\mathrm{T}}^{\text {miss }}$ threshold of $60 \mathrm{GeV}$ is passed. The following event selection cuts are applied:

- Pass standard data-quality requirements and combined photon plus $E_{\mathrm{T}}^{\text {miss }}$ trigger;

- Good primary vertex with at least 5 associated tracks;

- $E_{\mathrm{T}}^{\text {miss }}>50 \mathrm{GeV}$;

- Photon with $p_{T}>40 \mathrm{GeV}$; Only unconverted photons are considered to reduce the background from electrons mis-reconstructed as photons, if more than one such photon is found, the highest $p_{\mathrm{T}}$ photon is defined as signal photon candidate;

- To loosely select the VBF signature, at least two jets are required with $p_{T}>40 \mathrm{GeV}$ and at least one jet pair with $m_{j j}>400 \mathrm{GeV}$, where $m_{j j}$ is the invariant mass of the two jets and $\left|\Delta \eta_{j j}\right|>3.0$, with $\left|\Delta \eta_{j j}\right|$ being the magnitude of the separation of two jets in $\eta$;

- Jet veto: $\leq 1$ jet between the VBF jets since there is less radiation between VBF jets in signal than in background;

- VBF jet $p_{T} \geq 40 \mathrm{GeV}$;

- A lepton veto is applied for the signal region, validation region and most of the control regions to reduce electroweak backgrounds;

- Events are also vetoed if they have more than one selected non-VBF jet with $p_{\mathrm{T}}>30 \mathrm{GeV}$ and $\eta$ in the range spanned by the VBF jets;

- To reduce the backgrounds from $\gamma+$ jets and multi-jet events, the following cuts on the azimuthal angle are applied: $\mid\left.\Delta \phi\left(E_{\mathrm{T}}^{\text {miss }}, \mathrm{VBF}\right.$ jet $)\right|_{\min }>1.4$ and $\mid\left.\Delta \phi\left(E_{\mathrm{T}}^{\text {miss }}\right.$, non-VBF jet $)\right|_{\min }<$ 2.0.

Following this selection, the phase space is divided into 5 regions of phase space: a signal region, a validation region and three control regions for the combined $\gamma+$ jets and multi-jet background estimate. The regions B and D (see Figure 2) based on the angle between the photon and $E_{\mathrm{T}}^{\mathrm{miss}}$ and the kinematic quantities that distinguish VBF production $\left(m_{j j}\right.$ and $\left|\Delta \eta_{j j}\right|$, shown in Figure 3. To define regions A (signal region) and C, two additional cuts are applied:

- To reject non-VBF like events, an outside photon veto (OPV) cut is applied by requiring the photon to be between VBF jets in $\eta$;

- Vector sum $p_{\mathrm{T}}$ of all jets and photons is required to be $\geq 50 \mathrm{GeV}$.

Figure 4a shows the $\left|\Delta \phi\left(E_{\mathrm{T}}^{\text {miss }}, \gamma\right)\right|$ distribution in which the electroweak background is almost flat compared to the $\gamma+$ jets and multi-jet event are mostly in the high $\left|\Delta \phi\left(E_{\mathrm{T}}^{\text {miss }}, \gamma\right)\right|$ region. Figure $4 \mathrm{~b}$ shows the $E_{\mathrm{T}}^{\text {miss }}$ in the validation region, where the signal is concentrated at low $E_{\mathrm{T}}^{\text {miss }}$ values.

\section{Background estimation and systematic uncertainties}

A summary of backgrounds and estimation methods is given in table 1, the dominant backgrounds are normalised to CRs in data. The systematic uncertainties have been calculated on the 


\begin{tabular}{c|c|c}
\hline \hline Background & Distributions & Normalisation \\
\hline $\mathrm{W}(\rightarrow e v)$ & $\mathrm{W}(\rightarrow e v) \mathrm{MC}$ with $e \rightarrow \gamma$ misidentification rate from data & Data CR \\
$\mathrm{W} / \mathrm{Z}+$ jets & $\mathrm{W} / \mathrm{Z}+$ jets MC with jet $\rightarrow \gamma$ misidentification rate from MC & Data CR \\
$\mathrm{W} \gamma / \mathrm{Z} \gamma$ & $\mathrm{MC}$ & Data CR \\
Top and di-boson & $\mathrm{MC}$ & $\mathrm{MC}$ \\
$\gamma+$ jets and multijet & Data CR & Data CR \\
\hline \hline
\end{tabular}

Table 1: Summary of backgrounds and estimation methods. The dominant backgrounds are normalised to control regions (CR) in data [1].

number of events for the $\gamma+E_{\mathrm{T}}^{\text {miss }}$ signal and background in the signal region. The largest contributing factors for the signal region are the trigger efficiency uncertainty, jet energy resolution, jet energy scale, photon energy resolution and scale and the uncertainty from the signal cross section.

\section{Results and Interpretation}

The expected number of signal events in the signal region, assuming $\mathrm{BF}(h \rightarrow \mathrm{NLSP}+\mathrm{LSP})=$ $10 \%$ is shown in Figure 5. The best fit value of the signal yield corresponds to a BF of $11.6 \% \pm$ $10.9 \%$. The significance of the excess of observed data over the expected SM backgrounds is $1.1 \sigma$.

Figure 6 shows the expected and observed limits on the $\left(\sigma / \sigma_{\mathrm{SM}}\right) \times \mathrm{BF}(h \rightarrow \mathrm{NLSP}+\mathrm{LSP})$ at 95\% confidence level (CL) for the mono-photon and and di-photon signal samples, obtained using the CLs method [6] with an Asimov approximation. The $\pm 1 \sigma$ and $\pm 2 \sigma$ excursions of the expected limit in absence of signal are shown for all model parameters considered. Figure 7 shows the observed limits for different NLSP and LSP masses. Although the analysis was optimised for a $\gamma+E_{\mathrm{T}}^{\mathrm{miss}}$ search, strong upper limits are obtained on the branching fraction of the Higgs-boson to NLSP+LSP particles leading to a $\gamma \gamma+E_{\mathrm{T}}^{\text {miss }}$ final state.

\section{Conclusion}

A search for Higgs-bosons decaying to neutralinos and gavitinos with a final state signature

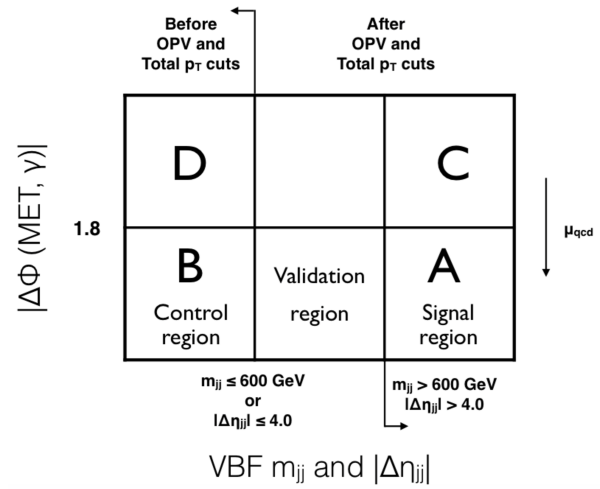

Figure 2: Division of phase space for the combined $\gamma+$ jets and multi-jet background estimate. 


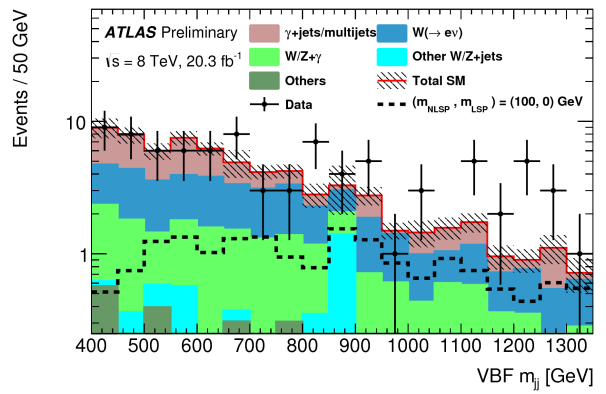

(a)

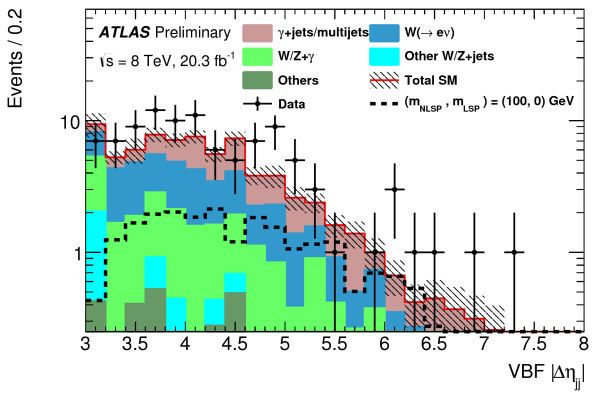

(b)

Figure 3: (a) VBF $m_{j j}$ and (b) $\operatorname{VBF}\left|\Delta \eta_{j j}\right|$ distributions after all requirements in the combined signal and validation regions [1].

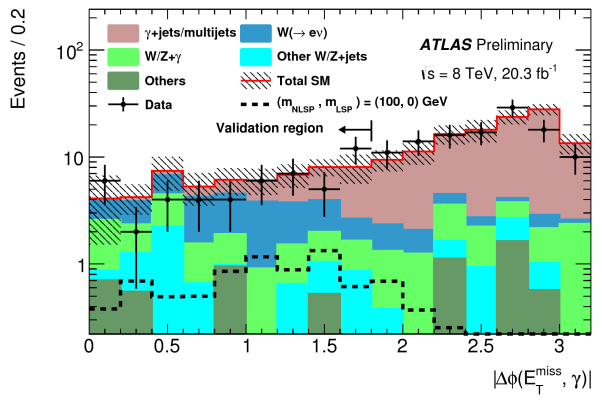

(a)

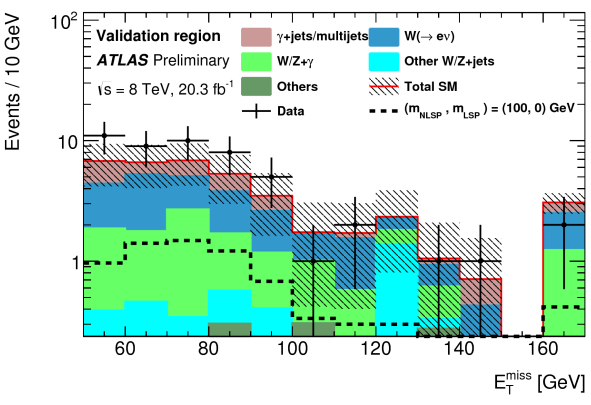

(b)

Figure 4: (a) The $\left|\Delta \phi\left(E_{\mathrm{T}}^{\text {miss }}, \gamma\right)\right|$ distribution with the requirement on it removed and (b) the $E_{\mathrm{T}}^{\text {miss }}$ distribution in the validation region [1].

of at least one photon, $E_{\mathrm{T}}^{\mathrm{miss}}$, and two VBF jets has been carried out, using the full $20128 \mathrm{TeV} p p$ dataset recorded by the ATLAS detector at the LHC, corresponding to an integrated luminosity of $20.3 \mathrm{fb}^{-1}$. The analysis was optimised for the $h \rightarrow \tilde{\chi}^{0} \tilde{G} \rightarrow \gamma \tilde{G} \tilde{G}$ signal leading to a final state of $\gamma+$ $E_{\mathrm{T}}^{\text {miss }}+j j$, but also applied to models with $h \rightarrow \tilde{\chi}_{2}^{0} \tilde{\chi}_{1}^{0} \rightarrow \gamma \gamma \tilde{\chi}_{1}^{0} \tilde{\chi}_{1}^{0}$ leading to a $\gamma \gamma+E_{\mathrm{T}}^{\text {miss }}+j j$ final state. After all selections, the number of observed events are in agreement with the SM backgrounds within uncertainties. The obtained limits are similar to or stronger than indirect limited from the Higgs-boson coupling measurements, and are the first direct limits on these Higgs-boson decays.

\section{References}

[1] ATLAS Collaboration, ATLAS-CONF-2015-001.

[2] ATLAS Collaboration, JINST 3 (2008) S08003.

[3] The GEANT4 Collaboration, S. Agostinelli et al., Nucl. Instrum. Meth. A506 (2003) 250.

[4] ATLAS Collaboration, Eur. Phys. J. C 70 (2010) 823-874.

[5] ATLAS Collaboration, ATL-PHYS-PUB-2010-013.

[6] A. L. Read, Journal of Physics G: Nuclear and Particle Physics 28 no. 10, (2002) 2693. 


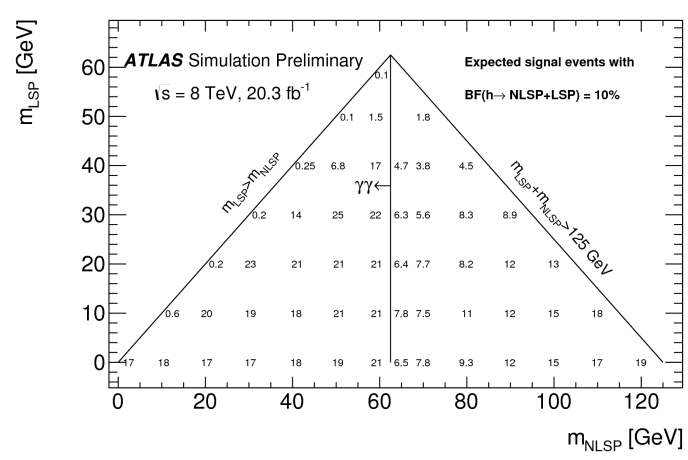

Figure 5: Expected number of signal events (assuming BF $(h \rightarrow$ NLSP + LSP $)=10 \%)$ in the signal region for different NLSP and LSP masses. The vertical line separates signal final states of $\gamma \gamma+E_{\mathrm{T}}^{\text {miss }}$ (left) from $\gamma+E_{\mathrm{T}}^{\text {miss }}$ (right) [1].

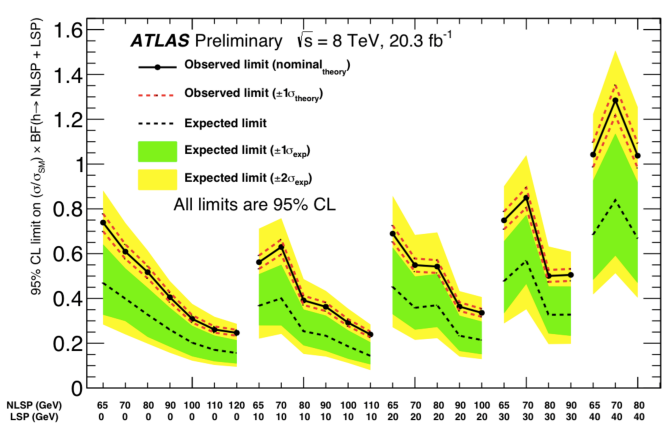

(a)

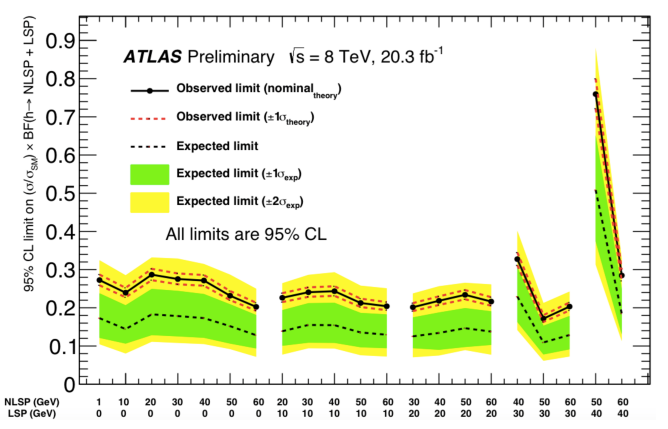

(b)

Figure 6: Observed and expected limits for various NLSP and LSP masses for (a) mono-photon signals and (b) di-photon signals. The green and yellow bands show the $\pm 1 \sigma$ and $\pm 2 \sigma$ excursions of the expected limits respectively [1].

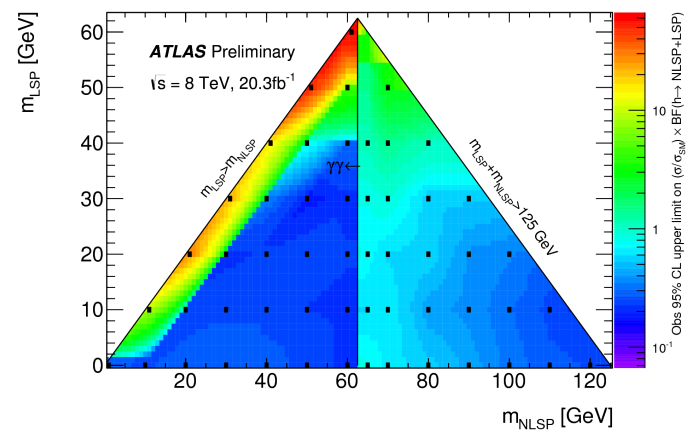

Figure 7: Observed limits for various NLSP and LSP masses. The black squares indicate the generated signal points that are used for interpolation [1]. 